\title{
Spectroscopic Study of the $B^{1} \Pi$ State of $\mathrm{NaH}$
}

Chia-Ching Chu, ${ }^{1}$ Chun Huang, ${ }^{1}$ Chin-Chun Tsai, ${ }^{2,} *$, and Thou-Jen Whang ${ }^{1, *}$,

${ }^{1}$ Department of Chemistry, National Cheng Kung University, Tainan 70101, Taiwan

${ }^{2}$ Department of Physics, National Cheng Kung University, Tainan 70101, Taiwan

${ }^{*}$ Corresponding author 
Table S1. The observed transitions.

(a) $e$ sublevels ( $P$ and $R$ lines)

\begin{tabular}{ccccc}
$B(0, J) \leftarrow\left(0, J^{\prime \prime}\right)$ & $\begin{array}{c}\text { Transition energy } \\
/ \mathrm{cm}^{-1}\end{array}$ & $J$ & $\begin{array}{c}\text { Transition energy } \\
/ \mathrm{cm}^{-1}\end{array}$ & $B\left(0, J \leftarrow X\left(1, J^{\prime \prime}\right)\right.$ \\
\hline$R\left(J^{\prime \prime}\right)=R(0)$ & 32034.2382 & 1 & 30900.3259 & $R(0)$ \\
$P(2)$ & 32005.3257 & 1 & 30873.3091 & $P(2)$ \\
$R(1)$ & 32031.8781 & 2 & 30899.2749 & $R(1)$ \\
$P(3)$ & 31984.9543 & 2 & 30853.2919 & $P(3)$ \\
$R(2)$ & 32025.0051 & 3 & 30892.3019 & $R(2)$ \\
$P(4)$ & 31957.5604 & 3 & 30827.4034 & $P(4)$ \\
$R(3)$ & 32012.2927 & 4 & 30880.1782 & $R(3)$ \\
$P(5)$ & 31925.4222 & 4 & 30795.7680 & $P(5)$ \\
$R(4)$ & 31993.2430 & 5 & 30862.6301 & $R(4)$ \\
$P(6)$ & 31887.2467 & 5 & 30759.8415 & $P(6)$ \\
$R(5)$ & 31967.8787 & 6 & 30838.7243 & $R(5)$ \\
$P(7)$ & 31842.8745 & 6 & 30716.9158 & $P(7)$ \\
$R(6)$ & 31936.8417 & 7 & 30809.2502 & $R(6)$ \\
$P(8)$ & 31792.9642 & 7 & 30669.4027 & $P(8)$ \\
$R(7)$ & 31898.1301 & 8 & 30772.1552 & $R(7)$ \\
$P(9)$ & 31735.5540 & 8 & 30613.8747 & $P(9)$
\end{tabular}

(b) $f$ sublevels ( $Q$ lines)

\begin{tabular}{ccccc}
$B(0, J) \leftarrow X\left(0, J^{\prime \prime}\right)$ & $\begin{array}{c}\text { Transition energy } \\
/ \mathrm{cm}^{-1}\end{array}$ & $J$ & $\begin{array}{c}\text { Transition energy } \\
/ \mathrm{cm}^{-1}\end{array}$ & $B(0, J) \leftarrow X\left(1, J^{\prime \prime}\right)$ \\
\hline$Q(1)$ & 32024.5949 & 1 & 30892.3019 & $Q(1)$ \\
$Q(2)$ & 32013.8300 & 2 & 30880.5599 & $Q(2)$ \\
$Q(3)$ & 31996.3140 & 3 & 30864.7272 & $Q(3)$ \\
$Q(4)$ & 31973.7048 & 4 & 30843.3882 & $Q(4)$ \\
$Q(5)$ & 31945.0035 & 5 & 30815.9958 & $Q(5)$ \\
$Q(6)$ & 31909.8356 & 6 & 30781.8235 & $Q(6)$ \\
$Q(7)$ & 31869.5642 & 7 & 30744.1323 & $Q(7)$ \\
$Q(8)$ & 31821.3929 & 8 & 30697.2894 & $Q(8)$
\end{tabular}

Table S2. FCFs and predicted transition bands $(\mathrm{NaH})$. NaH X to B [Huang et al. JCP(2010)133,044301 - Aymar et al. Can.J.Phys.(2009)87,543]. For each transition, 
the upper numeral shows the FCFs and the lower numeral shows the transition wavelength in Angstrom.

\begin{tabular}{|c|c|c|c|}
\hline \multicolumn{4}{|c|}{ FCFs $(* 10000)$} \\
\hline $\mathrm{X}(\mathrm{v}) \backslash \mathrm{B}(\mathrm{v})$ & 0 & 1 & 2 \\
\hline \multirow{2}{*}{0} & 29 & 24 & 8 \\
\hline & 3140 & 3130 & 3126 \\
\hline \multirow{2}{*}{1} & 115 & 83 & 27 \\
\hline & 3256 & 3245 & 3241 \\
\hline \multirow{2}{*}{2} & 288 & 179 & 56 \\
\hline & 3376 & 3365 & 3360 \\
\hline \multirow{2}{*}{3} & 534 & 278 & 82 \\
\hline & 3502 & 3489 & 3484 \\
\hline \multirow{2}{*}{4} & 803 & 335 & 90 \\
\hline & 3631 & 3618 & 3613 \\
\hline \multirow{2}{*}{5} & 1028 & 318 & 74 \\
\hline & 3766 & 3751 & 3746 \\
\hline \multirow{2}{*}{6} & 1161 & 235 & 43 \\
\hline & 3906 & 3890 & 3884 \\
\hline \multirow{2}{*}{7} & 1188 & 124 & 13 \\
\hline & 4050 & 4033 & 4027 \\
\hline \multirow{2}{*}{8} & 1119 & 33 & 0 \\
\hline & 4199 & 4181 & 4174 \\
\hline \multirow{2}{*}{9} & 981 & 0 & 10 \\
\hline & 4353 & 4333 & 4326 \\
\hline \multirow{2}{*}{10} & 809 & 45 & 42 \\
\hline & 4511 & 4490 & 4482 \\
\hline \multirow{2}{*}{11} & 632 & 167 & 87 \\
\hline & 4672 & 4649 & 4641 \\
\hline \multirow{2}{*}{12} & 469 & 348 & 130 \\
\hline & 4836 & 4812 & 4803 \\
\hline \multirow{2}{*}{13} & 331 & 564 & 158 \\
\hline & 5002 & 4976 & 4967 \\
\hline \multirow{2}{*}{14} & 222 & 791 & 160 \\
\hline & 5169 & 5141 & 5131 \\
\hline \multirow{2}{*}{15} & 140 & 1004 & 130 \\
\hline & 5333 & 5303 & 5293 \\
\hline \multirow{2}{*}{16} & 80 & 1177 & 72 \\
\hline & 5491 & 5460 & 5449 \\
\hline
\end{tabular}




$\begin{array}{rrrr}17 & 41 & 1276 & 9 \\ & 5640 & 5607 & 5596 \\ 18 & 16 & 1248 & 32 \\ & 5774 & 5739 & 5727 \\ 19 & 4 & 1035 & 400 \\ & 5885 & 5849 & 5836 \\ 20 & 0 & 605 & 1777 \\ & 5966 & 5929 & 5916 \\ 21 & 0 & 117 & 5037 \\ & 6011 & 5974 & 5961\end{array}$

Table S3. FCFs and predicted transition bands $(\mathrm{NaD}) . \mathrm{NaD} \mathrm{X}$ to $\mathrm{B}[\mathrm{Chu}$ et al. JCP(2017)147,024301 - Aymar et al. Can.J.Phys.(2009)87,543(using the reduced-mass of $\mathrm{NaD}$ for solving the Hamiltonian with the $\mathrm{NaH}$ potential energy curve)]. For each transition, the upper numeral shows the FCFs and the lower numeral shows the transition wavelength in Angstrom.

\begin{tabular}{|c|c|c|c|c|}
\hline \multicolumn{5}{|c|}{$\operatorname{FCFs}(* 10000)$} \\
\hline$X(v) \backslash B(v)$ & 0 & 1 & 2 & 3 \\
\hline \multirow{2}{*}{0} & 5 & 8 & 5 & 2 \\
\hline & 3126 & 3117 & 3113 & 3110 \\
\hline \multirow{2}{*}{1} & 28 & 37 & 25 & 11 \\
\hline & 3210 & 3200 & 3195 & 3193 \\
\hline \multirow{2}{*}{2} & 89 & 103 & 65 & 27 \\
\hline & 3295 & 3285 & 3279 & 3277 \\
\hline \multirow{2}{*}{3} & 205 & 204 & 119 & 48 \\
\hline & 3383 & 3372 & 3366 & 3364 \\
\hline \multirow{2}{*}{4} & 376 & 316 & 166 & 63 \\
\hline & 3473 & 3462 & 3456 & 3453 \\
\hline \multirow{2}{*}{5} & 581 & 395 & 182 & 65 \\
\hline & 3566 & 3554 & 3548 & 3545 \\
\hline \multirow{2}{*}{6} & 784 & 408 & 157 & 51 \\
\hline & 3661 & 3648 & 3642 & 3639 \\
\hline \multirow{2}{*}{7} & 947 & 346 & 100 & 27 \\
\hline & 3759 & 3746 & 3739 & 3736 \\
\hline \multirow{2}{*}{8} & 1045 & 233 & 40 & 7 \\
\hline & 3859 & 3845 & 3838 & 3835 \\
\hline \multirow{2}{*}{9} & 1065 & 110 & 3 & 0 \\
\hline & 3962 & 3947 & 3940 & 3936 \\
\hline
\end{tabular}




\begin{tabular}{|c|c|c|c|c|}
\hline \multirow{2}{*}{10} & 1014 & 22 & 7 & 9 \\
\hline & 4068 & 4052 & 4044 & 4041 \\
\hline \multirow{2}{*}{11} & 908 & 1 & 52 & 32 \\
\hline & 4176 & 4159 & 4151 & 4147 \\
\hline \multirow{2}{*}{12} & 771 & 57 & 119 & 56 \\
\hline & 4286 & 4269 & 4260 & 4256 \\
\hline \multirow{2}{*}{13} & 624 & 178 & 184 & 73 \\
\hline & 4399 & 4380 & 4371 & 4367 \\
\hline \multirow{2}{*}{14} & 483 & 341 & 224 & 75 \\
\hline & 4513 & 4494 & 4485 & 4480 \\
\hline \multirow{2}{*}{15} & 359 & 514 & 226 & 61 \\
\hline & 4630 & 4610 & 4600 & 4595 \\
\hline \multirow{2}{*}{16} & 256 & 672 & 190 & 38 \\
\hline & 4749 & 4727 & 4717 & 4712 \\
\hline \multirow{2}{*}{17} & 175 & 792 & 126 & 13 \\
\hline & 4868 & 4846 & 4835 & 4830 \\
\hline \multirow{2}{*}{18} & 115 & 861 & 56 & 0 \\
\hline & 4989 & 4965 & 4953 & 4948 \\
\hline \multirow{2}{*}{19} & 72 & 874 & 7 & 7 \\
\hline & 5109 & 5085 & 5072 & 5067 \\
\hline \multirow{2}{*}{20} & 42 & 836 & 7 & 39 \\
\hline & 5229 & 5204 & 5190 & 5185 \\
\hline \multirow{2}{*}{21} & 23 & 754 & 81 & 95 \\
\hline & 5347 & 5321 & 5307 & 5301 \\
\hline \multirow{2}{*}{22} & 12 & 639 & 247 & 161 \\
\hline & 5463 & 5435 & 5420 & 5414 \\
\hline \multirow{2}{*}{23} & 5 & 504 & 510 & 216 \\
\hline & 5573 & 5544 & 5529 & 5523 \\
\hline \multirow{2}{*}{24} & 2 & 364 & 857 & 226 \\
\hline & 5678 & 5648 & 5632 & 5625 \\
\hline \multirow{2}{*}{25} & 0 & 233 & 1241 & 161 \\
\hline & 5774 & 5742 & 5726 & 5719 \\
\hline \multirow{2}{*}{26} & 0 & 124 & 1562 & 36 \\
\hline & 5858 & 5826 & 5810 & 5802 \\
\hline \multirow{2}{*}{27} & 0 & 48 & 1647 & 53 \\
\hline & 5929 & 5896 & 5879 & 5872 \\
\hline \multirow{2}{*}{28} & 0 & 10 & 1289 & 929 \\
\hline & 5982 & 5948 & 5931 & 5924 \\
\hline
\end{tabular}




$\begin{array}{rrrrr}29 & 0 & 0 & 478 & 3816 \\ 6015 & 5981 & 5964 & 5956\end{array}$

Table S4. Dunham fitting (e sublevels, NaH).

\begin{tabular}{|c|c|c|c|c|}
\hline $\mathrm{V}$ & $\mathrm{J}$ & OBS & CALC & $\mathrm{O}-\mathrm{C}$ \\
\hline 0 & 1 & 32616.1969 & 32615.9158 & 0.2811 \\
\hline 0 & 2 & 32624.7708 & 32624.0258 & 0.745 \\
\hline 0 & 3 & 32635.9333 & 32636.083 & -0.1497 \\
\hline 0 & 4 & 32651.9308 & 32651.9583 & -0.0275 \\
\hline 0 & 5 & 32671.4307 & 32671.4792 & -0.0485 \\
\hline 0 & 6 & 32694.2261 & 32694.4303 & -0.2042 \\
\hline 0 & 7 & 32720.9205 & 32720.553 & 0.3675 \\
\hline 0 & 8 & 32749.4894 & 32749.5459 & -0.0565 \\
\hline 0 & 1 & 32616.1402 & 32615.9158 & 0.2244 \\
\hline 0 & 2 & 32623.4392 & 32624.0258 & -0.5866 \\
\hline 0 & 3 & 32635.8763 & 32636.083 & -0.2067 \\
\hline 0 & 4 & 32652.1092 & 32651.9583 & 0.1509 \\
\hline 0 & 5 & 32671.6159 & 32671.4792 & 0.1367 \\
\hline 0 & 6 & 32694.3873 & 32694.4303 & -0.043 \\
\hline 0 & 7 & 32721.0257 & 32720.553 & 0.4727 \\
\hline 0 & 8 & 32749.4817 & 32749.5459 & -0.0642 \\
\hline 0 & 1 & 32616.5091 & 32615.9158 & 0.5933 \\
\hline 0 & 2 & 32624.6513 & 32624.0258 & 0.6255 \\
\hline 0 & 3 & 32636.2709 & 32636.083 & 0.1879 \\
\hline 0 & 4 & 32651.4599 & 32651.9583 & -0.4984 \\
\hline 0 & 5 & 32671.6338 & 32671.4792 & 0.1546 \\
\hline 0 & 6 & 32694.0364 & 32694.4303 & -0.3939 \\
\hline 0 & 7 & 32721.0235 & 32720.553 & 0.4705 \\
\hline 0 & 8 & 32749.1039 & 32749.5459 & -0.442 \\
\hline 0 & 1 & 32615.3421 & 32615.9158 & -0.5737 \\
\hline 0 & 2 & 32623.6884 & 32624.0258 & -0.3374 \\
\hline 0 & 3 & 32635.5019 & 32636.083 & -0.5811 \\
\hline 0 & 4 & 32651.5376 & 32651.9583 & -0.4207 \\
\hline 0 & 5 & 32671.4976 & 32671.4792 & 0.0184 \\
\hline 0 & 6 & 32694.4162 & 32694.4303 & -0.0141 \\
\hline 0 & 7 & 32721.0425 & 32720.553 & 0.4895 \\
\hline 0 & 8 & 32749.2758 & 32749.5459 & -0.2701 \\
\hline
\end{tabular}

FITTED DUNHAM CONSTANTS 


\begin{tabular}{cccc}
$\mathrm{Y}(00)$ & $.3261387935 \mathrm{D}+05$ & $\mathrm{DY}(00)$ & $.1459028359 \mathrm{D}+00$ \\
$\mathrm{Y}(01)$ & $.2038265209 \mathrm{D}+01$ & $\mathrm{DY}(01)$ & $.1093317921 \mathrm{D}-01$ \\
$\mathrm{Y}(02)$ & $-.1795338097 \mathrm{D}-02$ & $\mathrm{DY}(02)$ & $.1511601203 \mathrm{D}-03$ \\
\hline SIGFIT & $.3913213205 \mathrm{D}+00$ & &
\end{tabular}

Table S5. Dunham fitting (f sublevels, $\mathrm{NaH}$ ).

\begin{tabular}{|c|c|c|c|c|}
\hline $\mathrm{v}$ & $\mathrm{J}$ & OBS & CALC & $\mathrm{O}-\mathrm{C}$ \\
\hline 0 & 1 & 32616.156 & 32616.2534 & -0.0974 \\
\hline 0 & 2 & 32624.7012 & 32624.3091 & 0.3921 \\
\hline 0 & 3 & 32636.1305 & 32636.2907 & -0.1602 \\
\hline 0 & 4 & 32652.0777 & 32652.0756 & 0.0021 \\
\hline 0 & 5 & 32671.5121 & 32671.5005 & 0.0116 \\
\hline 0 & 6 & 32694.0196 & 32694.3613 & -0.3417 \\
\hline 0 & 7 & 32720.9158 & 32720.4129 & 0.5029 \\
\hline 0 & 8 & 32749.3492 & 32749.3697 & -0.0205 \\
\hline 0 & 1 & 32616.7154 & 32616.2534 & 0.462 \\
\hline 0 & 2 & 32623.7599 & 32624.3091 & -0.5492 \\
\hline 0 & 3 & 32636.0866 & 32636.2907 & -0.2041 \\
\hline 0 & 4 & 32652.2557 & 32652.0756 & 0.1801 \\
\hline 0 & 5 & 32671.6877 & 32671.5005 & 0.1872 \\
\hline 0 & 6 & 32693.6158 & 32694.3613 & -0.7455 \\
\hline 0 & 7 & 32721.2529 & 32720.4129 & 0.84 \\
\hline 0 & 8 & 32748.9102 & 32749.3697 & -0.4595 \\
\hline \multicolumn{5}{|c|}{ FITTED DUNHAM CONSTANTS } \\
\hline $\mathrm{Y}(00)$ & .326 & $091 \mathrm{D}+05$ & $\mathrm{DY}(00)$ & $.2383215003 \mathrm{D}+00$ \\
\hline $\mathrm{Y}(01)$ & .202 & $030 \mathrm{D}+01$ & $\mathrm{DY}(01)$ & .1785854027D-01 \\
\hline $\mathrm{Y}(02)$ & -.17 & $5258 \mathrm{D}-02$ & $\mathrm{DY}(02)$ & $.2469088859 \mathrm{D}-03$ \\
\hline
\end{tabular}

Table S6. Dunham fitting (all levels, $\mathrm{NaH}$ ).

\begin{tabular}{ccccc}
$\mathrm{v}$ & $\mathrm{J}$ & OBS & CALC & O-C \\
\hline 0 & 1 & 32616.1969 & 32616.0283 & 0.1686 \\
0 & 2 & 32624.7708 & 32624.1202 & 0.6506 \\
0 & 3 & 32635.9333 & 32636.1522 & -0.2189 \\
0 & 4 & 32651.9308 & 32651.9974 & -0.0666 \\
0 & 5 & 32671.4307 & 32671.4863 & -0.0556
\end{tabular}




\begin{tabular}{|c|c|c|c|c|}
\hline 0 & 6 & 32694.2261 & 32694.4073 & -0.1812 \\
\hline 0 & 7 & 32720.9205 & 32720.5063 & 0.4142 \\
\hline 0 & 8 & 32749.4894 & 32749.4872 & 0.0022 \\
\hline 0 & 1 & 32616.1402 & 32616.0283 & 0.1119 \\
\hline 0 & 2 & 32623.4392 & 32624.1202 & -0.681 \\
\hline 0 & 3 & 32635.8763 & 32636.1522 & -0.2759 \\
\hline 0 & 4 & 32652.1092 & 32651.9974 & 0.1118 \\
\hline 0 & 5 & 32671.6159 & 32671.4863 & 0.1296 \\
\hline 0 & 6 & 32694.3873 & 32694.4073 & -0.02 \\
\hline 0 & 7 & 32721.0257 & 32720.5063 & 0.5194 \\
\hline 0 & 8 & 32749.4817 & 32749.4872 & -0.0055 \\
\hline 0 & 1 & 32616.5091 & 32616.0283 & 0.4808 \\
\hline 0 & 2 & 32624.6513 & 32624.1202 & 0.5311 \\
\hline 0 & 3 & 32636.2709 & 32636.1522 & 0.1187 \\
\hline 0 & 4 & 32651.4599 & 32651.9974 & -0.5375 \\
\hline 0 & 5 & 32671.6338 & 32671.4863 & 0.1475 \\
\hline 0 & 6 & 32694.0364 & 32694.4073 & -0.3709 \\
\hline 0 & 7 & 32721.0235 & 32720.5063 & 0.5172 \\
\hline 0 & 8 & 32749.1039 & 32749.4872 & -0.3833 \\
\hline 0 & 1 & 32615.3421 & 32616.0283 & -0.6862 \\
\hline 0 & 2 & 32623.6884 & 32624.1202 & -0.4318 \\
\hline 0 & 3 & 32635.5019 & 32636.1522 & -0.6503 \\
\hline 0 & 4 & 32651.5376 & 32651.9974 & -0.4598 \\
\hline 0 & 5 & 32671.4976 & 32671.4863 & 0.0113 \\
\hline 0 & 6 & 32694.4162 & 32694.4073 & 0.0089 \\
\hline 0 & 7 & 32721.0425 & 32720.5063 & 0.5362 \\
\hline 0 & 8 & 32749.2758 & 32749.4872 & -0.2114 \\
\hline 0 & 1 & 32616.156 & 32616.0283 & 0.1277 \\
\hline 0 & 2 & 32624.7012 & 32624.1202 & 0.581 \\
\hline 0 & 3 & 32636.1305 & 32636.1522 & -0.0217 \\
\hline 0 & 4 & 32652.0777 & 32651.9974 & 0.0803 \\
\hline 0 & 5 & 32671.5121 & 32671.4863 & 0.0258 \\
\hline 0 & 6 & 32694.0196 & 32694.4073 & -0.3877 \\
\hline 0 & 7 & 32720.9158 & 32720.5063 & 0.4095 \\
\hline 0 & 8 & 32749.3492 & 32749.4872 & -0.138 \\
\hline 0 & 1 & 32616.7154 & 32616.0283 & 0.6871 \\
\hline 0 & 2 & 32623.7599 & 32624.1202 & -0.3603 \\
\hline 0 & 3 & 32636.0866 & 32636.1522 & -0.0656 \\
\hline
\end{tabular}




\begin{tabular}{ccrrl}
0 & 4 & 32652.2557 & 32651.9974 & 0.2583 \\
0 & 5 & 32671.6877 & 32671.4863 & 0.2014 \\
0 & 6 & 32693.6158 & 32694.4073 & -0.7915 \\
0 & 7 & 32721.2529 & 32720.5063 & 0.7466 \\
0 & 8 & 32748.9102 & 32749.4872 & -0.577 \\
\hline \multicolumn{5}{c}{ FITTED DUNHAM CONSTANTS } \\
Y(00) & $.3261399653 \mathrm{D}+05$ & $\mathrm{DY}(00)$ & $.1243304502 \mathrm{D}+00$ \\
$\mathrm{Y}(01)$ & $.2033559150 \mathrm{D}+01$ & $\mathrm{DY}(01)$ & $.9316659848 \mathrm{D}-02$ \\
$\mathrm{Y}(02)$ & $-.1763950484 \mathrm{D}-02$ & $\mathrm{DY}(02)$ & $.1288104217 \mathrm{D}-03$ \\
\hline $\mathrm{SICIT}$ & $.4084067514 \mathrm{D}+00$ &
\end{tabular}

